\title{
Didactique de l'anglais commercial : de l'usage d'un exemple, hypermarché et supercentre
}

\section{Dominique Claisse}

\section{(2) OpenEdition}

\section{Journals}

Édition électronique

URL : http://journals.openedition.org/asp/4077

DOI : 10.4000/asp.4077

ISSN : 2108-6354

\section{Éditeur}

Groupe d'étude et de recherche en anglais de spécialité

\section{Édition imprimée}

Date de publication : 1 décembre 1994

Pagination : 177-182

ISSN : 1246-8185

\section{Référence électronique}

Dominique Claisse, « Didactique de l'anglais commercial : de l'usage d'un exemple, hypermarché et supercentre », ASp [En ligne], 5-6 | 1994, mis en ligne le 11 décembre 2013, consulté le 02 mai 2019 URL : http://journals.openedition.org/asp/4077 ; DOI : 10.4000/asp.4077

Ce document a été généré automatiquement le 2 mai 2019.

Tous droits réservés 


\title{
Didactique de l'anglais commercial : de l'usage d'un exemple, hypermarché et supercentre
}

\author{
Dominique Claisse
}

1 L'étudiant en Techniques de commercialisation, filière commerciale des IUT, reçoit un enseignement spécialisé et apprend à connaître un monde professionnel où abondent les termes anglo-saxons. L'enseignement de l'anglais, langue de spécialité, permet souvent de préciser le sens des termes employés et les notions qu'ils recouvrent. L'enseignant assume alors un rôle nouveau: poursuite de l'approfondissement des connaissances au travers de l'apprentissage de la langue de spécialité et mise en lumière des différentes formes de culture de consommation et/ou de distribution, mais aussi, dans une féconde relation d'échanges avec l'apprenant en cours de spécialisation dans le domaine qui sert de support à l'étude de la langue, sensibilisation à la fois à l'existence d'une forme d'altérité et réflexion commune sur les modes de fonctionnement et de pensée du monde professionnel dans lequel l'apprenant évoluera bientôt: à la fois enrichissement interculturel et approfondissement intraculturel, au travers de l'étude d'une langue différente, abordée sous l'angle technologique.

2 En effet, cette démarche interactive entre l'enseignant et l'apprenant permet souvent à ce dernier de réfléchir à sa propre culture professionnelle, précise certaines des notions dominantes dans le domaine dans lequel il se spécialise - notions qui sont pour beaucoup d'origine américaine - et apporte un éclairage différent et complémentaire sur le comportement des professionnels, modelé, souvent à leur insu, outre-Atlantique.

Le commerce de détail français montre dans sa langue tous les signes d'une forte influence linguistique anglo-saxonne; l'apprenant en prend vite conscience, tant au travers de l'enseignement qu'il reçoit qu'auprès des professionnels qu'il côtoie. Ce simple constat empirique mérite toutefois une certaine attention. Un essai de lecture cursive pratiqué sur deux exemplaires de Libre-Service Actualités, l'un des deux principaux magazines français dans ce domaine ( $\mathrm{N}^{\circ} 1379,6$ janvier 1994 et $\mathrm{N}^{\circ} 1381,20$ janvier 1994), 
permet de relever 239 occurrences de termes anglo-saxons. La plupart de ces termes sont utilisés sans guillemets ni italiques, sans explications non plus, un peu comme si leur sens était directement accessible au lecteur. On ne sera pas surpris de trouver 38 fois le terme marketing, 13 fois merchandising (marchandisage), 9 fois management (gestion; management, prononcé à la française est cependant accepté officiellement aujourd'hui), 16 fois discount (minimarge). Certains termes techniques récents apparaissent au fil des pages: si facing (frontale) et couponing (couponnage) ne posent guère de problèmes à l'apprenant, plus déroutants peuvent être les termes self-scanning (auto-encaissement: opération de passage en caisse et enregistrement des marchandises effectués par le client lui-même), sourcing (approvisionnement, politique d'approvisionnement) ou encore rackjobbing (pratique commerciale qui consiste pour un fabricant à disposer et à gérer ses propres gondoles de présentation de marchandises dans une surface de vente, avec l'aval $\mathrm{du}$ distributeur). Les néologismes, souvent tentatives maladroites pour franciser certains termes anglo-saxons, ne sont pas rares: c'est le cas en l'espèce de pratique " discountrice » et d'élément de présentation « pegboardable ».

4 Ces quelques exemples mettent en lumière certains phénomènes de nature linguistique : ils sont autant de signes d'un besoin langagier que la langue spécialisée de l'apprenant ne satisfait qu'imparfaitement, d'où ces emprunts plus ou moins bien assimilés. Ils sont aussi souvent source de confusion: le même terme en anglais et en français recouvre parfois des réalités ou notions différentes. Que doit-on comprendre du terme marketing en français ? Il est souvent difficile de distinguer entre mercatique (qui relève du domaine de la recherche: analyse du comportement du consommateur, détection des besoins, adéquation entre production et consommation), marchéage (étude des différents paramètres du plan de marchéage, connu sous le nom de marketing mix: product, place, price et promotion), commercialisation ou encore publicité. De même, packaging signifie-til conditionnement, emballage ou habillage (mise en valeur) du produit? Ils sont aussi le signe d'une forte résistance face aux incitations qui sont faites à utiliser des traductions qui, pour imparfaites qu'elles paraissent à certains, ont au moins le mérite d'être plus facilement compréhensibles pour l'apprenant moyen : au cours de cette lecture, si l'on a pu lire les termes mailing, merchandising ou cash and carry par exemple, les traductions équivalentes proposées par le "Dictionnaire des termes officiels" (Journal officiel de la République Française) n'ont pas été relevées, à savoir respectivement publipostage, marchandisage ou payer-prendre. Enfin, beaucoup de ces termes empruntés montrent la gêne ou le malaise linguistique qui apparait lorsqu'il s'agit de transposer en français la forme en -ing. Mais ce phénomène n'est-il que linguistique ? La forme en -ing est aussi en l'occurrence synonyme de souplesse, symbole peut-être de progression, de progrès donc.

5 L'enseignement de l'anglais de spécialité permet déjà de mettre en relief ce foisonnement d'emprunts linguistiques et de poser une question plus importante encore : ces emprunts ne sont-ils qu'un épiphénomène, une mode ou une tendance passagère, ou sont-ils au contraire autant de signes d'une empreinte plus profonde, de nature culturelle cette fois?

6 Il n'y a pas dépendance économique du monde commercial français. Les grands groupes français sont à capitaux français. L'impact culturel, en revanche, semble fort, et aider l'apprenant à en déceler les signes fait aussi partie de la tâche de l'enseignant d'anglais de spécialité

7 Les rappels historiques entrent mal dans le cadre d'une didactique qui se veut interactive. On peut se contenter de mentionner la période qui commence après la Deuxième Guerre mondiale et les missions de productivité qui ont aussi touché le monde commercial 
français. On peut toutefois noter que l'apprenant n'aura pas, dans le cadre de son enseignement spécialisé, l'occasion d'aborder cette perspective historique. C'est pourtant à partir de cette époque que le commerce s'apprend et s'enseigne sous la forme que l'on connaît aujourd'hui, et les techniques que l'étudiant découvre se nomment plus volontiers management, marketing, merchandising ou discount que techniques de gestion, mercatique, marchandisage ou minimarge.

8 La mercatique est à la base de cet enseignement technique spécialisé ; on peut à ce sujet avancer une hypothèse: l'activité intellectuelle professionnelle connue sous le nom de marketing est peut-être la traduction en termes commerciaux de la philosophie américaine, le Pragmatisme.

9 Les parallèles sont nombreux en effet: si l'on admet que le Pragmatisme se compose de quatre veines essentielles, on peut tenter de retrouver la traduction en termes commerciaux de chacune d'entre elles. C'est ainsi que l'empirisme radical représenté par William James suggère la théorie des essais et erreurs (trials and errors), le sens de l'expérience, la prédominance de ce qui est efficace, et ces méthodes nouvelles s'opposent alors à la vision ancienne du commerce européen, marqué par l'immobilisme, le sens du luxe et du décorum, symbolisé par les lois cadenas de l'avant-guerre. La veine scientifique de Charles Pierce annonce la pratique des études et enquêtes, le recours aux techniques quantitatives de gestion et s'oppose totalement à cette autre conception qui voudrait que le commerce soit un art, fondé sur des qualités innées. La réalité dans ce domaine est probablement à mi-chemin entre ces deux extrêmes mais le recours aux mathématiques, à la statistique ou à l'analyse des données a représenté à l'origine un changement radical de perspective. Comment ne pas évoquer encore cette forme de Darwinisme économique, dont le représentant pourrait être Georges Mead, et qui parle de renouvellement ou d'évolution des formes commerciales et se distingue là aussi du commerce institutionnalisé et des traditions des grands magasins, héritées d'une génération à l'autre durant des décennies. Enfin l'idéal démocratique, dont le meilleur avocat pourrait être John Dewey, trouve peut-être sa traduction dans le supermarché, que l'on peut imaginer sous la forme d'une corne d'abondance ouverte au plus grand nombre (Cornucopia devenue réalité, en quelque sorte). Cet aspect est bien éloigné des accusations dont le commerce moderne a longtemps souffert en Europe : celle de traiter différemment les clients selon leur niveau de fortune ou, pire encore, le soupçon de parasitisme que l'on sent encore présent de temps à autre.

10 Aborder ces divers aspects de façon concrète, au travers de dossiers composés à partir de documents spécialisés authentiques et récents dans les deux langues permet d'ouvrir des horizons nouveaux. L'analyse contrastive de deux formules de vente proches, l'hypermarché en Europe et le supercenter aux États-Unis, venant en conclusion d'un de ces dossiers en est un exemple. Cette synthèse, qui va mener à l'étude tour à tour de l'hypermarché puis du supercenter, est construite de façon à proposer, à partir de phénomènes linguistiques précis et au travers de l'étude de la réalité commerciale, une interprétation de nature culturelle. La démarche adoptée conduira à ce qui est l'objet essentiel de cet article, l'enseignement de l'anglais de spécialité à des apprenants qui se forment aux matières commerciales.

11 L'hypermarché est souvent appelé en France l'hypermarché «à la française »; on parlera même à son sujet de concept franco-français. Le terme est français, créé en 1966 à Paris, par un groupe de professionnels de l'Institut français du Libre Service et de LSA. On l'appelait jusqu'alors magasin discount, voire parfois cargo. Le terme hypermarché a 
suscité des critiques : hyper est l'équivalent en grec du latin super, bien que supermarché et hypermarché soient des formules commerciales très différentes par nature (le supermarché propose surtout des produits alimentaires, à la différence de l'hypermarché qui offre en outre des articles textiles, de bazar, des produits blancs ou bruns). Au-delà des critiques, ces quelques faits montrent, outre la volonté d'affirmer une personnalité propre, une forme de réticence à admettre ou accepter une influence américaine jugée trop forte. La recherche permet alors de mettre en lumière quelques éléments révélateurs: l'hypermarché est d'origine belge, sous influence américaine. Créé en septembre 1961 à Auderghem, près de Bruxelles, par Maurice Cauwe (le premier hypermarché français a été créé en 1963 par Marcel Fournier et la maison Carrefour, à Sainte-Geneviève-des-Bois près de Paris), il s'appelait alors self-service checkout one-level discount department store with food and parking. Maurice Cauwe était disciple et ami proche de Bernardo Trujillo, américain d'origine colombienne, reconnu dans le monde entier comme le maître à penser du commerce de détail moderne. La dérision dont faisaient preuve les professionnels français à l'endroit de M. Cauwe quand ils le surnommaient le "Cauwe boy » en dit long, à la fois sur l'impact du commerce à l'américaine et l'attitude qui prévalait alors en France face à cette influence.

On est alors fondé à parler à l'apprenant de phénomène commercial européen, battant ainsi en brèche une forme d'esprit hexagonal. La prise de conscience d'une identité européenne du commerce de détail passe là aussi par les mots ; Euro-retailing se rencontre ainsi de plus en plus fréquemment dans la presse spécialisée anglo-saxonne. Un bref retour aux sources de cette influence permettra d'enrichir des notions commerciales telles que discount, value (rapport qualité-prix) et merchandising. Les techniques marchandes propres à l'hypermarché évoquent en effet une forme de culture, celle du client-roi dans une société de consommation que l'on dit souvent être "à l'américaine ".

L'hypermarché présente également des spécificités qui vont permettre à l'apprenant de mieux comprendre sa propre culture commerciale. L'affirmation de cette personnalité apparait par exemple au travers du «tout-sous-le-même-toit», ou aptitude de l'hypermarché à réunir en un seul lieu les cinq rayons, un peu comme les cinq doigts de la main : les produits de grande consommation (PGC), les produits frais, les articles textiles, le blanc (électro-ménager) et le brun (audio-visuel, radio, ordinateurs par exemple). Cette capacité à mettre ensemble, à centraliser ou fusionner, fait de la consommation un acte unique, en un lieu qui devient une sorte de cathédrale commerciale. Refusé au début par les Américains, ce concept sera peu à peu adapté. On parlera en anglais à l'origine de allunder-one-roof, aujourd'hui de one-stop shopping convenience.

Une anecdote met en lumière une autre différence entre les deux cultures professionnelles. Les consommateurs américains ont reproché à l'hypermarché de ne pas avoir de bagging boys (ensacheurs), et ce simple fait permet d'évoquer alors une autre culture de consommation. Le service est en effet perçu de façon différente sur les deux continents, le consommateur américain étant infiniment plus puissant, donc plus exigeant, que son homologue européen. Un autre point enfin révèle un aspect professionnel non négligeable : le rôle de chef de rayon en Europe s'apparente à celui d'un petit chef d'entreprise, alors que le chef de rayon américain voit souvent son activité restreinte à celle d'un animateur; le grand commerce américain montre là une vision plus centralisatrice de la gestion (on doit toutefois noter à ce sujet l'évolution en cours du rôle du chef de rayon en France, qui tend à se rapprocher de celui que l'on connaît outreAtlantique). 
15 L'hypermarché apparaît alors comme une démarche commerciale globale et le terme qu'on lui attribue parfois, celui de commerce " déspécialisé », dit bien la différence avec les formes américaines de grand commerce, caractérisées par la spécialisation et la division nette et précise des formules proposées. L'hypermarché « à l'européenne ", fier de ses succès, a tenté de traverser l'Atlantique afin d'y conquérir de nouveaux marchés et d'acquérir, selon les dires de certains professionnels, ses lettres de noblesse. Des implantations ont eu lieu entre 1985 et 1992 : Euromarché, puis Carrefour, Auchan et Leclerc ont tenté l'aventure. Toutes ces implantations ont échoué. Mais l'événement en tant que tel n'a d'intérêt pour l'enseignant d'anglais de spécialité dans sa relation avec l'apprenant que dans la mesure où il est l'occasion d'analyser les raisons de l'échec et les formes prises par la réponse américaine à cette tentative de pénétration.

16 Les causes qui expliquent pourquoi la greffe de l'hypermarché aux États-Unis n'a pas réussi sont diversement analysées par les spécialistes. Elles sont parfois commerciales : choix d'une période difficile pour l'ensemble du commerce américain; difficultés de trouver un positionnement clair entre prix et service ; l'hypermarché était plus cher que le discount department store et ses prestations en matière de service étaient très inférieures à celle du supermarché ; gigantisme enfin. Elles sont aussi culturelles : les mentalités sont "différentes », dit-on parfois sommairement. Alors que l'Européen regroupe, centralise en quelque sorte, l'Américain préférerait une séparation nette entre les différentes familles de produits proposées. Il est vrai dans ce domaine que l'association de produits alimentaires et textiles dans un même magasin a surpris de nombreux Américains; ces derniers en revanche ne sont guère étonnés de voir cohabiter les produits alimentaires et les produits pharmaceutiques et d'hygiène (les health and beauty care products, connus sous l'acronyme $\mathrm{HBC}$ ).

17 Un petit détail, de nature linguistique celui-ci, peut apporter un éclairage nouveau. Il semble qu'il y ait eu aux États-Unis une sorte de malaise dans la dénomination de cette formule de vente. C'est ainsi que l'on trouve fréquemment les termes hypermarket, hypermart, hypermarche (sans accent), et le très révélateur hybrid market: ce type de magasin, hybride donc, ni véritablement minimarge, ni entièrement supermarché, a surpris et désorienté. Enfin l'association phonétique possible avec le terme hype (substantif et verbe; hype: intensive or exaggerated publicity or sales promotion), ou son intensif overhype, ravive par trop ce soupçon de parasitisme déjà évoqué. Le distributeur américain, soucieux d'œuvrer davantage dans une industrie de services que dans ce qu'il est convenu d'appeler le commerce, ne pouvait qu'être réticent devant l'emploi de ce terme. Le consommateur, lui, fort de ses nombreux succès remportés depuis des années grâce à des associations puissantes et efficaces, peut avoir perçu cette formule de vente comme un possible retour en arrière. Détail linguistique peut-être, mais aux effets évocateurs désastreux dans ce cas, et qui montre s'il en est besoin l'importance du verbe, dont l'usage ne doit rien laisser au hasard.

18 La riposte américaine a suivi un processus en trois étapes, qui sont toutes riches de connotations culturelles. Professionnalisme d'abord, quand les deux plus grandes chaînes américaines, inquiètes de cette arrivée de l'hypermarché, ont accepté de dépenser des sommes considérables afin d'analyser le concept européen. Réalisme et capacité à emprunter et à assimiler les points forts des concurrents ensuite, quand Wal-Mart et KMart ont décidé d'ouvrir eux aussi quelques hypermarchés afin d'en mettre les qualités à l'essai. Souplesse et aptitude à américaniser encore quand, après un repli sur les deux formules de vente les plus populaires aux États-Unis, à savoir le discount department store 
(magasin minimarge, spécialisé dans les produits bazar et $\mathrm{HBC}$ ) et le supermarché (spécialisé en produits alimentaires), ils ont créé une nouvelle formule de vente, connue à l'origine sous le nom riche de sens de combination store. Transformer un discount department store en combination store revenait en fait à intégrer au magasin minimarge un supermarché alimentaire, les deux parties de cette nouvelle formule de vente étant bien sûr très nettement séparées. Le processus modulaire ainsi adopté trouve d'ailleurs son expression également au travers des termes. Deux modules de base permettent en effet d'aborder la plupart des dénominations des formules commerciales américaines. L'un, store, évoque des magasins aussi divers que les célèbres drugstores ou les department stores, mais aussi le superstore (grand supermarché en américain, petit hypermarché en GrandeBretagne) ou le warehouse store (magasin-entrepôt) par exemple. L'autre, center, est lui aussi riche de combinaisons possibles: on peut évoquer ici le strip center (centre d'unités commerciales indépendantes), l'ethnic center (centre ethnique: destiné plus particulièrement aux membres d'une même minorité), le power center enfin (centre d'énergie, qui regroupe plusieurs magasins minimarges ou tueurs de catégorie, les category killers). SuperCenter a d'abord été nom propre, associé à combination store, sous la forme SuperCenter combination store, et devient bien vite en 1993 le supercenter, pour des raisons de commodité probablement. L'atout essentiel de l'hypermarché est préservé, à savoir la notion de one-stop shopping convenience; il n'y a pas fusion mais association de produits alimentaires, de bazar et d'hygiène (les produits textiles ont été définitivement retirés). L'élément prix et le rapport qualité-prix (value) redeviennent les armes essentielles qui permettent de se battre pour de nouvelles parts de marché. Les premières ouvertures viennent d'avoir lieu mais le succès est tel qu'on prévoit l'ouverture de plusieurs centaines de supercentres, aux États-Unis et ailleurs.

19 L'importance des termes choisis, où l'approximation n'a pas sa place, mais aussi la façon de répondre à la menace, de s'approprier les atouts des concurrents, de faire preuve de réalisme et d'efficacité sont autant de sources d'enseignement pour l'apprenant au plan commercial. La vision américaine ainsi mise en lumière autorise peut-être à parler alors, au-delà des phénomènes culturels et linguistiques, d'enrichissement commercial et professionnel au travers de l'étude de la langue de spécialité.

20 En retour, la réaction européenne ne manque pas d'intérêt. Déçus de leur échec, les distributeurs européens considèrent le supercenter comme une pâle copie de l'hypermarché, un magasin terne et ennuyeux, sans harmonie, tout en en reconnaissant l'excellence en matière de gestion et d'organisation. La notion d'art de vivre est parfois perçue de façon si différente des deux côtés de l'Atlantique!

21 L'objectif essentiel de cette analyse était de présenter une des nombreuses façons d'aborder l'enseignement de l'anglais de spécialité à des apprenants d'une filière technologique, dans ce cas, Techniques de commercialisation. L'analyse linguistique de termes spécialisés, utilisés à la fois par les professionnels ou les enseignants de matières techniques, termes présents également dans des documents anglo-saxons, permet de comparer ou de préciser l'usage qui en est fait, de les dépouiller aussi parfois d'une aura de nature quasi affective qui en obscurcit le sens. L'approche culturelle est l'occasion de sensibiliser l'apprenant aux influences exercées ou subies et d'élargir peut-être la vision commerciale : les notions d'interdépendance et de fertilisation croisée s'imposent vite.

Mettre en parallèle l'hypermarché et le supercenter mène à une prise de conscience des différences: les mots sont proches, mais ne sont pas équivalents (comment traduire supercenter ou hypermarché ?); les réalités commerciales non plus. Il n’y a pas de 
correspondance exacte : les nuances qui apparaissent sont peut-être alors ce qui forme la culture, véritable prisme au travers duquel sont lues ou relues ces mêmes réalités commerciales, selon les goûts et affinités de chaque peuple. Confronter ainsi des formules de vente, puis plus tard des notions plus subtiles (proximité et convenience par exemple) peut être une des nombreuses façons d'aborder de façon efficace l'enseignement de la langue de spécialité.

\section{BIBLIOGRAPHIE}

Cauwe, M. 1985. La Rage de grandir, La rage de grandir, ou Historique et chronique d'un petit Bazar à la grande entreprise aux multiples enseignes, 1882-1982.

Drewbear, P. 1970. Mass Merchandising Revolution and Evolution. New York : Fairchild Publication Inc.

McGoldrick, P. 1990. Retail Marketing. Londres : Mcgraw-Hill Company.

Uhrich, R. 1962. Supermarchés et usines de distribution, hier aux États-Unis, aujourd'hui en France. Paris : Plon.

Tordjman, A. 1987. Viewpoints on the Changing Consumer Scene. Marketing Science Institute Conference.

Tordjman, A. 1988. Le Commerce de détail américain. Paris : Les Éditions d'organisation.

Revues américaines

Progressive Grocer

Stores

Discount Merchandiser

\section{Revues britanniques}

Retail Week

Supermarketing

Grocer

\section{Revues françaises}

Libre-Services-Actualités

Points de Vente

\section{RÉSUMÉS}

L'enseignement de l'anglais de spécialité appliqué au commerce de détail permet de mettre en relief les signes d'une forte influence américaine. L'analyse comparée de l'hypermarché et du supercentre est un moyen de préciser ressemblances et spécificités. Tous deux évoquent le pragmatisme; l'un est généraliste, l'autre est modulaire. Leur étude linguistique et culturelle conduit peut-être à une meilleure prise de conscience de phénomènes commerciaux. 
Teaching English for Special Purposes when applied to retailing stresses signs of a strong American influence. The comparative analysis of the Hypermarket and the Supercenter is a way to emphasize various similarities and specificities. Both suggest Pragmatism; one is comprehensive, the other is modular. The linguistic and cultural approach might lead to a better apprehension of commercial phenomena.

INDEX

Mots-clés : anglais du commerce, commerce de détail, hypermarché, pragmatisme

Keywords : English for commerce, hypermarket, pragmatism, retailing

\section{AUTEUR}

\section{DOMINIQUE CLAISSE}

Dominique Claisse enseigne à l'IUT de Valenciennes. dominique.claisse@univ-valenciennes.fr 